\title{
Stage IB Eyelid Carcinoma AJCC v7
}

National Cancer Institute

\section{Source}

National Cancer Institute. Stage IB Eyelid Carcinoma A/CC V7. NCI Thesaurus. Code C88122.

Stage IB includes: T2a, N0, M0. T2a: Tumor more than $5 \mathrm{~mm}$, but not more than $10 \mathrm{~mm}$ in greatest dimension. Or, any tumor that invades the tarsal plate or eyelid marg in. NO: No regional lymph node metastasis. M0: No distant metastasis. (AJCC 7th ed.) 